\title{
Critical layer thickness of MOVPE-grown GaAs on $\operatorname{In}_{x} \mathrm{Ga}_{1-x}$ As
}

\author{
J. te Nijenhuis, P.J. van der Wel, R.W.F. van Asten, P.R. Hageman and L.J. Giling \\ Department of Experimental Solid State Physics III, RIM, Faculty of Science, University of Nijmegen, Toernooiveld, 6525 ED Nijmegen, \\ The Netherlands
}

\begin{abstract}
In this study we report investigations of strain and relaxation in MOVPE-grown GaAs layers on In-alloyed GaAs LEC substrates, with an In-content between $0.1 \%$ and $1.15 \%$. The experimentally determined critical layer thicknesses for the formation of misfit dislocations in the GaAs layers are compared with theoretical calculations of the Gibbs free energy of the epilayer, containing a half loop dislocation which has released a certain amount of elastic energy. A very good matching between theory and experiments is obtained, even in the range of very small misfits. The shift of the bandgap energy as induced by the strain in the epilayer is given by the deformation potential theory. These shifts have been measured using photoluminescence at liquid He temperatures. It appears from these measurements that for layers grown beyond the critical thickness the amount of relaxation is exactly determined by the theoretical equilibrium between elastical deformation and the formation of misfit dislocations. In the region of low misfit dislocation density the dislocations can be distinguished separately by high spatial resolution photoluminescence. When the density becomes too high the depletion regions around the dislocations are overlapping each other. The individual dislocations are not discernable anymore.
\end{abstract}

\section{Introduction}

An important consequence of heteroepitaxial growth of III-V compound semiconductors is the lattice mismatch, which is generated by a difference in lattice constant between the substrate and the epilayer. In thin layers the misfit will be accommodated by elastical deformation of the epilayer. The growth of the layer is pseudomorphic, the lattice of the epilayer parallel to the interface will be strained in order to fit the substrate lattice. When the thickness of the layer is larger than a certain critical layer thickness, the growth is not pseudomorphic anymore, the strained layer will relax by formation of misfit dislocations in the interface. Both the strain and the misfit dislocations have a strong influence on the electrical and optical material properties [1-4].

To study the effect of strain and relaxation, one usually has a substrate with a fixed lattice constant and epilayers with varying chemical compositions and lattice constants. For instance, in the systems $\operatorname{In}_{x} \mathrm{Ga}_{1-x} \mathrm{P}$ grown on GaAs by VPE [1] and LPE [2] and in $\operatorname{In}_{x} \mathrm{Ga}_{1-x}$ As on InP variation of the electro-optical properties is induced by the changed chemical composition as well as by the strain. However, in these systems there are two intrinsic disadvantages. (i) One has to know the composition of the grown layer exactly in order to separate the contribution of the strain and of the misfit dislocations to the change of the material properties from the contribution of the alloy. (ii) The physical and mechanical parameters of the totally relaxed alloys are not always known, so one has to use approximate values, obtained from interpolation by assuming linear behaviour, for a comparison between theory and experiments.

In our studies on heteroepitaxial systems, we have avoided these difficulties by using (100) $\mathrm{In}_{x} \mathrm{Ga}_{1-x} \mathrm{As}$ substrates with known amounts of indium (between $0.1 \%$ and $1.15 \%$ ) upon which a layer of undoped $\mathrm{GaAs}$ is grown. This gives rise to a maximum lattice mismatch between the $\mathrm{In}_{x} \mathrm{Ga}_{1-x}$ As substrate and the GaAs epilayer of $8 \times 10^{-4}$. In this system, the uncertainty of the chemical composition of the epilayer is eliminated, because a binary compound semiconductor is grown instead of a ternary or even a quaternary alloy. Therefore the chemical composition of the epilayer is exactly known. Changes in morphology and electro-optical properties of the epilayer compared with conventional GaAs homoepitaxy are 
now only a consequence of the strain and the misfit dislocations in the epilayer and not of the changed chemical composition.

In the literature, the growth of GaAs by MBE and MOVPE on In-alloyed GaAs is mentioned [5-10]. However, a precise determination of the critical layer thickness of epitaxially grown GaAs is not given. The aim of this paper is to give a comparison between the experimentally found amount of strain in the epitaxial layer and the critical layer thickness for GaAs as given by theory. Further, the spatial distribution of misfit dislocations is studied with defect revealing DSL etching and high spatial resolution photoluminescence, which shows the local effect of misfit dislocations on the photoluminescence signal.

\section{Experimental}

In our experiments we used (100) $\operatorname{In}_{x} \mathrm{Ga}_{1-x}$ As LEC substrates [11] as obtained from LETI, Grenoble, France. The amounts of indium in the substrates were measured by inductively-coupled plasma atomic emission spectroscopy (ICPAES), from which the In concentration in the substrates was known with an accuracy of $10^{-4}$. The local variation in In content in these substrates is ca. $1-2 \%$ [11]. The dislocation density is $\leq 10^{4} \mathrm{~cm}^{-2}$ [12].

Epitaxial layers of undoped GaAs have been grown upon these substrates in a conventional low pressure MOVPE reactor system. Trimethylgallium (TMG) and arsine were used as precursors. The ratio of arsine and TMG (the V/III ratio) at the entrance of the reactor was 125 . The growth temperature was $913 \mathrm{~K}$. The growth rate was ca. $1.6 \mu \mathrm{m} / \mathrm{h}$. For a comparison with $\mathrm{GaAs}$ homoepitaxy (100) $2^{\circ}$ (110) horizontal Bridgman (HB) GaAs substrates, as obtained from MCP (UK), were also placed in the reactor in the same growth experiments.

The morphology of the grown layers was observed with an interference-contrast microscope. The thickness of the layers was measured in a scanning electron microscope (SEM) after cleaving and etching.
To determine the band gap energy, photoluminescence experiments were carried out at $4 \mathrm{~K}$ with an energy resolution of $0.1 \mathrm{meV}$ around the spectral range of the band gap of GaAs. Shifts of the exciton spectrum were measured by a comparison with the (unstrained) $\mathrm{GaAs}$ spectrum.

Several layers also have been examined by local photoluminescence at $4.2 \mathrm{~K}$ with a spatial resolution of $1 \mu \mathrm{m}$. Details about the photoluminescence microscope are described elsewhere [13]. The microscopic differences around a dislocation influence the photoluminescence intensity either in a positive or in a negative way. This results in bright and dark regions on the PL intensity mapping. These PL intensity mappings, recorded at the wavelength of the acceptor bound exciton, are compared with the (defect revealing) DSL etch patterns [14], from which the individual dislocations are recognized. The interesting regions are more explicitly analysed by taking PL spectra at fixed positions.

\section{Results and discussion}

The relative lattice mismatch $f$ between the epilayer and the subtrate is defined as:

$f=\left(a_{\mathrm{s}}-a_{0}\right) / a_{0}$,

in which $a_{\mathrm{s}}$ is the lattice constant of the substrate and $a_{0}$ the lattice constant of the totally relaxed epilayer. The relative strain in the epilayer $\epsilon$ is defined in the same way as:

$\epsilon=\left(a_{\mathrm{e}}-a_{0}\right) / a_{0}$,

in which $a_{\mathrm{e}}$ is the lattice constant of the strained epilayer. From this definition it follows that the strain is positive for a system which is under tensile stress. In the case the misfit is accommodated only by elastical deformation, $a_{\mathrm{e}}$ equals $a_{\mathrm{s}}$, and therefore $f=\epsilon$. Samples grown under this condition show a specular surface (fig. 1a). When the layers are grown beyond a certain critical layer thickness, the misfit is also accommodated by dislocations in the interface. The relation between the mismatch and the strain is now given by $f=\epsilon+\delta$, in which $\delta$ is the amount of plastical 

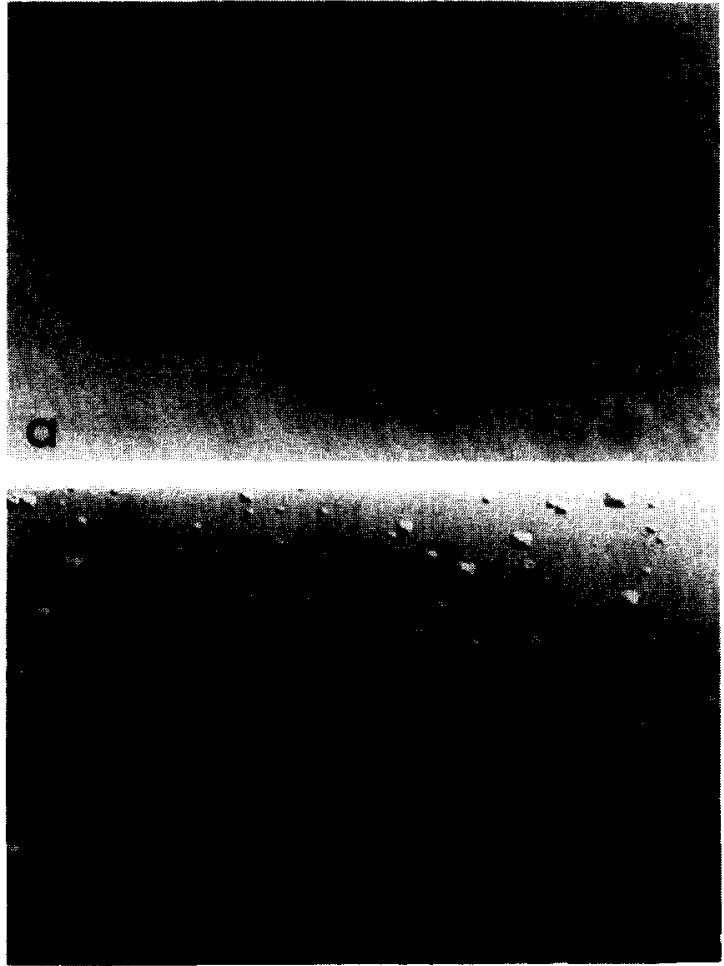

Fig 1. Surface morphologies of as-grown GaAs epilayers of (a) $0.4 \mu \mathrm{m}$ and (b) $4.4 \mu \mathrm{m}$ thickness on $\operatorname{In}_{0.008} \mathrm{Ga}_{0.992} \mathrm{As}$.

deformation. On the surface of these layers a cross hatched pattern is observed, as shown in fig. $1 \mathrm{~b}$.

To compare the observed critical layer thickness with theory, we have assumed spontaneous nucleation of half-loop dislocations in the epilayer. From calculations of the Gibbs free energy $G$ of a layer containing a half-loop dislocation, which has released a certain amount of elastic energy, the critical layer thickness is determined as a function of the lattice mismatch. This theory assumes that the misfit dislocations are $60^{\circ}$ dislocations along the $\langle 110\rangle$ directions in a $\{111\}$ glide plane in diamond-like crystals. Originally this theory was developed by Matthews [15] who calculated the critical layer thickness for which $G$ is at a maximum. Van de Leur et al. [16] introduced the energy balance model. In this theory the thickness for which $G=0$ is defined as the critical layer thickness. The results of the calculations for both theories are shown in fig. 2. In this figure also the results from the growth experiments are given, together with the results of Inoue et al. [5], although in this paper no growth conditions of the epilayer are mentioned, nor are the layer thicknesses measured precisely. It appears that layers containing misfit dislocations are lying above the theoretical curve, obtained from ref. [16], while the unrelaxed layers are lying below it. The energy balance model [16] gives a very good match between theory and experiment, even in the region with a very small misfit in which the elastic energy, i.e. "the driving force" for the formation of misfit dislocations, is very small.

The biaxial stress, as generated by the lattice mismatch, breaks up the degeneracy of the valence band [2], which shifts the effective bandgap energy. The calculated bandgap energy shifts for the light hole and the heavy hole valence band at $\boldsymbol{k}=0$ induced by strain are given by

$$
\begin{aligned}
& \Delta E_{\mathrm{lh}}=\left(2 a \frac{c_{11}-c_{12}}{c_{11}}-b \frac{c_{11}+c_{12}}{c_{11}}\right) \epsilon, \\
& \Delta E_{\mathrm{hh}}=\left(2 a \frac{c_{11}-c_{12}}{c_{11}}+b \frac{c_{11}+c_{12}}{c_{11}}\right) \epsilon,
\end{aligned}
$$

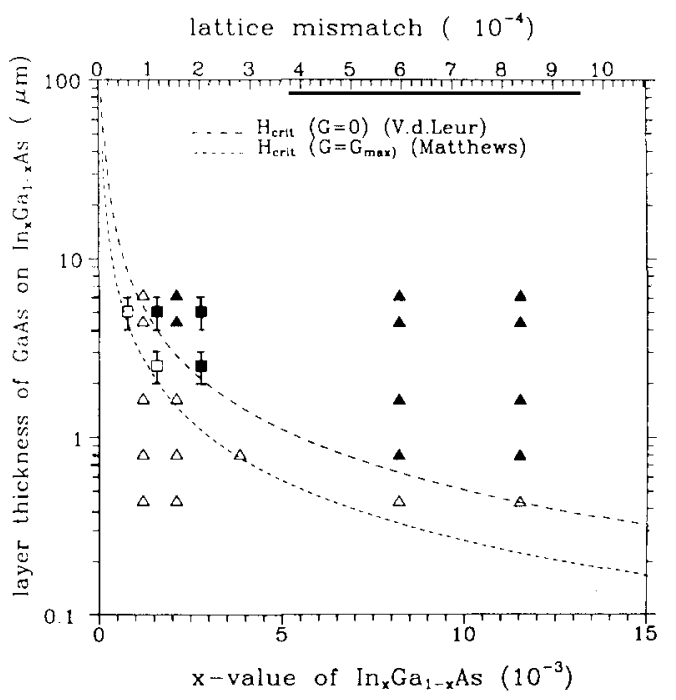

Fig 2. Calculated critical layer thickness as a function of In content in InGaAs (lower scale) and mismatch $f$ (upper scale), according to refs. [15] and [16]. Filled and open symbols indicate samples with and without misfit dislocations. Triangles are samples as described in this work. Squares are data obtained from ref. [5]. 


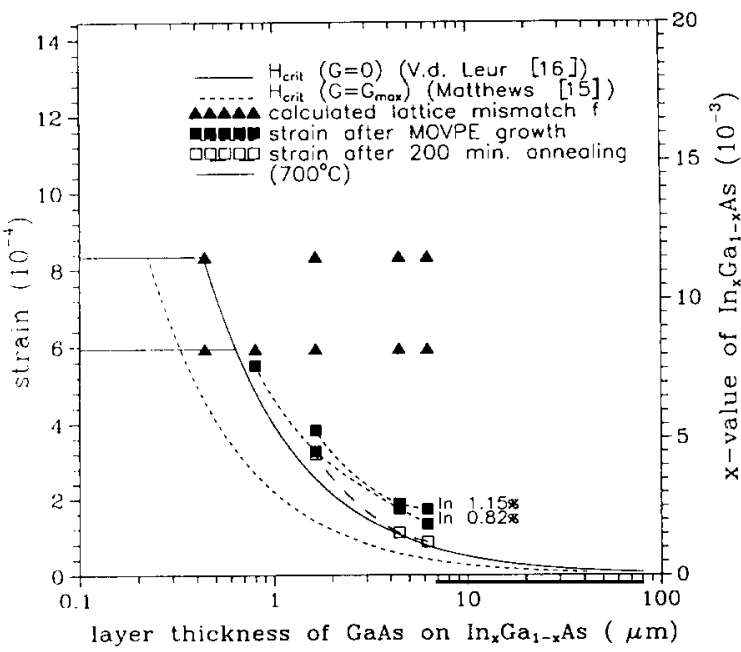

Fig 3. Strain in the epilayer as a function of layer thickness. For thin layers, which are not relaxed by misfit dislocations, the strain is equal to the lattice mismatch $f$. When the layer thickness is larger than the critical value, the misfit dislocations release the strain until it has reached the value of the mismatch for which the layer thickness is critical.

where $c_{i j}$ are the elastic stiffness coefficients, $a$ and $b$ are constants obtained from the deformation potential theory $[17,18]$. Using the values as given in refs. $[19,20]$, we calculate for GaAs

$\Delta E_{\mathrm{lh}}=-5.84 \epsilon(\mathrm{eV})$,

$\Delta E_{\mathrm{hh}}=-12.35 \epsilon(\mathrm{eV})$.

It follows that $\Delta E_{\mathrm{hh}}$ has a stronger shift than $\Delta E_{\mathrm{lh}}$. For systems which are under tensile stress, i.e. in the case that is positive, the bandgap will be between the conduction band and the heavy hole valence band. Because the bandgap energy is not directly measured in photoluminescence experiments, we assume that the shift of the exciton spectrum gives a good approximation for the shift of the bandgap. From this it is possible to determine the amount of relaxation in the epilayer. The resulting strain as measured from these photoluminescence experiments as a function of layer thickness is shown in fig. 3. For thin layers the strain $\epsilon$ is equal to the lattice mismatch $f$. For layers with thicknesses larger than the critical value, the strain has been relieved only partially. The residual strain of the layer equals the value of the mismatch for which the layer thickness is critical. Since the theory is developed for the formation of the first misfit dislocation, we conclude that in the observed region of very small misfits the nucleation process is not influenced by the presence of already existing misfit dislocations. Post-growth annealing for $200 \mathrm{~min}$ at $973 \mathrm{~K}$ under As pressure does hardly change the amount of relaxation. These results indicate that the GaAs layers already reach the predicted equilibrium during growth, which is in agreement with $\operatorname{In}_{x} \mathrm{Ga}_{1-x}$ As samples grown by MBE on GaAs [21,22].

The effect of the misfit dislocations in the epilayer on the luminescence intensity is investigated by photoluminescence microscopy. A typical PL intensity mapping is shown in fig. 4a. This
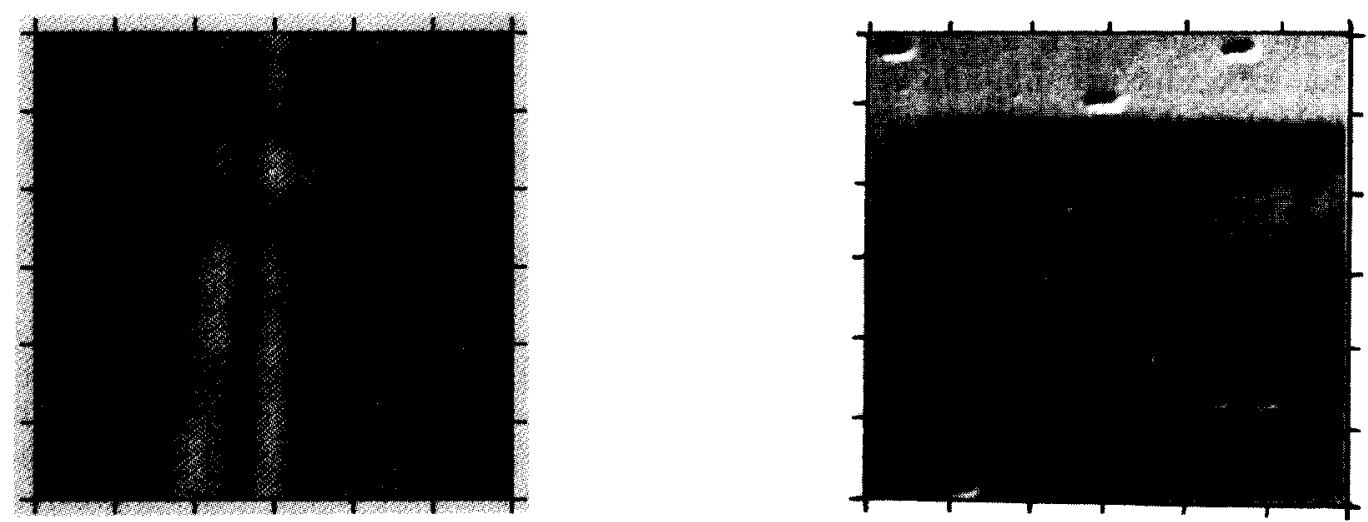

Fig. 4. (a) PL intensity mapping and (b) interference-contrast photomicrograph of the same area of a GaAs epilayer with a thickness of $4.4 \mu \mathrm{m}$ on $\mathrm{In}_{0.002} \mathrm{Ga}_{0.998}$ As after DSL etching. The bar indicates $25 \mu \mathrm{m}$ for both pictures. 
is the mapping of a layer of GaAs of $4.4 \mu \mathrm{m}$ thickness on $\mathrm{In}_{0.002} \mathrm{Ga}_{0.998} \mathrm{As}$, which contains misfit dislocations. The mapping can be compared with the etch pattern of the same area which is shown at the same scale as the mapping in fig. $4 \mathrm{~b}$. The dark lines on the mapping are also recognized on the micrograph. The PL spectra as measured on these lines have a tendency to shift to higher energy values with respect to the matrix material between the dislocations. One of these dark lines is decorated by a bright region. This region which is clearly seen on the mapping is not revealed on the etch pattern. On the other hand the facets as seen on the photograph cannot be distinguished by local photoluminescence.

As the core material around a misfit dislocation is deformed and contains an array of dangling bonds, which can act as non-radiative levels [4], the photoluminescence intensity on the dislocation is strongly decreased. However, the core of a dislocation has a typical radius of $10 \mathrm{~nm}$, which is much smaller than the typical linewidth of the dark regions of $10 \mu \mathrm{m}$. This can be explained by assuming that around a dislocation a depletion region is present. The free carriers, which are excited by the laser light, will diffuse to the dislocation where they can recombine on the nonradiative levels. Further, the shift of the PL spectra towards higher energy values on the dark regions indicates that the material around a dislocation is somewhat more relaxed than the material in the matrix. So the dark regions most probably contain one misfit dislocation, which is in accordance with the etch pattern.

The bright region around one of the dislocations is not yet completely understood. Probably here the quality of the material is higher, because of the diffusion of impurities and of native defects to the core of the dislocation. The facets on the etch pattern are not seen on the PL mapping, so there is no dislocation expected within a facet. The facets and the bright region will be the subject of future investigations.

From the density of the dark lines we can see that in this layer only a small part of the misfit is relaxed by the misfit dislocations. In the case of complete relaxation the average value of the distance between the dislocations in this epilayer should be $2 \mu \mathrm{m}$. When the density of misfit dislocations is increased, the dark regions overlap each other. This influences the electro-optical properties of the material severely. On the PL mapping a very clear difference between the dislocations and the matrix material is not observed anymore [23].

\section{Conclusions}

It is shown that the epilayer-substrate system with a fixed layer composition is a good system to observe the effects of the strain and relaxation on the electro-optical properties without any influence of change in chemical composition. The critical layer thickness for the formation of misfit dislocations in GaAs under tensile stress as experimentally found is in good agreement with the the energy balance model in the region of very small misfit. No complete relaxation is found after growth as well as after annealing. The remaining strain in the layers is also described by the equilibrium between elastical deformation and the formation of misfit dislocations. The nucleation of misfit dislocations is not influenced by the presence of existing dislocations.

A comparison of DSL etch patterns and PL intensity mapping shows that the electro-optical effect of the dislocation is much larger than only the plastically deformed core of the dislocation itself. From this it follows that misfit dislocations should be avoided as much as possible when constructing opto-electronical devices from heteroepitaxial systems.

\section{Acknowledgements}

The authors wish to thank Mrs. Dr. A. Chabli from LETI for kindly supplying the InGaAs substrates, Mr. S.M. Olsthoorn and Mr. F.A.J.M. Driessen for performing the photoluminescence experiments, and Mr. E.R.H. van Eck for assistance in the local photoluminescence experiments. This work is part of the research program of the Stichting FOM (Dutch Foundation for Fundamental Research on Matter) with financial sup- 
port from NWO (Dutch Organization for Scientific Research).

\section{References}

[1] G.H. Olsen, C.J. Nuese and R.T. Smith, J. Appl. Phys. 49 (1978) 5523.

[2] H. Asai and K. Oe, J. Appl. Phys. 54 (1983) 2052.

[3] C.P. Kuo, S.K. Vong, R.M. Cohen and G.B.Stringfellow, J. Appl. Phys. 57 (1985) 5428, and references therein.

[4] J.M. Woodall, G.D. Pettit, T.N. Jackson, C. Lanza, K.L. Kavanagh and J.W. Mayer, Phys. Rev. Letters 51 (1983) 1783.

[5] T. Inoue, S. Nishine, M. Shibata, T. Matsutomo, S. Yoshitake, Y. Sato, T. Shimoda and K. Fujita, in: Proc. 12th Intern. Symp. on. GaAs and Related Compounds, Karuizawa, 1985, Inst. Phys. Conf. Ser. 79, Ed. M. Fujimoto (Inst. Phys., London-Bristol, 1986) p. 7.

[6] M. Morioka, T. Mishima, K. Hiruma, Y. Katayama and Y. Shiraki, in: Proc. 12th Intern. Symp. on GaAs and Related Compounds, Karuizawa, 1985, Inst. Phys. Conf. Ser. 79, Ed. M. Fujimoto (Inst. Phys., London-Bristol, 1986) p. 121.

[7] M. Shinohara, T. Ito and Y. Imamura, J. Appl. Phys. 58 (1985) 3449.

[8] M. Shinohara, T. Ito, K. Yamada and Y. Imamura, Japan. J. Appl. Phys. 24 (1985) L711.

[9] H. Takeuchi, M. Shinohara and K. Oe, Japan. J. Appl. Phys. 24 (1986) L303.
[10] T, Imai, S. Fuke, K. Mori and K. Kuwahara, Appl. Phys. Letters 24 (1989) 816.

[11] E. Molva, Ph. Bunod, F. Roturier, A. Chabli, F. Bertin, J.P. Leludec and P. Larnaudie, in: Semi-Insulating III-V Materials, Malmö, 1988, Eds. G. Grossmann and L. Ledebo (Hilger, Bristol, 1988).

[12] A. Chabli, E. Molva, A. George, F. Bertin, P. Bunod and J. Blétry, in: Proc. European Mater. Res. Soc. Meeting, Strasbourg, 1986.

[13] E.P. Visser and L.J. Giling, Rev. Sci. Instr. 61 (1990).

[14] J.L. Weyher and J. van de Yen, J. Crystal Growth 63 (1983) 285.

[15] J.W. Matthews, in: Epitaxial Growth, Part B, Ed. J.W. Matthews (Academic Press, New York, 1975).

[16] R.H.M. van de Leur, A.J.G. Schellingerhout, F. Tuinstra and J.E. Mooij, J. Appl. Phys. 64 (1988) 3043.

[17] F.H. Pollak and M. Cardona, Phys. Rev. 172 (1968) 816.

[18] A. Gavini and M. Cardona, Phys. Rev. B1 (1970) 672.

[19] Landolt-Börnstein, Group III, Vol. 17a, Physics of the Group IV Elements and III-V Compounds (Springer, Berlin, 1982).

[20] A.R. Goñi, K. Strössner, K. Syassen and M. Cardona, Phys. Rev. B36 (1987) 1581.

[21] A.V. Drigo, A. Aydinli, A. Carnera, F. Genova, C. Rigo, C. Ferrari, P. Franzosi and G. Salviati, J. Appl. Phys. 66 (1989) 1975.

[22] P.M.J. Marée, J.C. Barbour, J.F. van der Veen, K.L. Kavanagh, C.W.T. Bulle-Lieuwma and M.P.A. Viegers, J. Appl. Phys. 62 (1987) 4413.

[23] J. te Nijenhuis, P.J. van der Wel, E.R.H. van Eck and L.J. Giling, unpublished results. 